\title{
BMJ Open The economics of physical activity in low- income and middle-income countries: protocol for a systematic review
}

\author{
Priyanga Diloshini Ranasinghe, ${ }^{1,2}$ Subhash Pokhrel, ${ }^{3}$ Nana Kwame Anokye ${ }^{3}$
}

To cite: Ranasinghe PD, Pokhrel S, Anokye NK. The economics of physical activity in low-income and middleincome countries: protocol for a systematic review. BMJ Open 2019;9:e022686. doi:10.1136/ bmjopen-2018-022686

- Prepublication history and additional material for this paper are available online. To view these files, please visit the journal online (http://dx.doi. org/10.1136/bmjopen-2018022686).

Received 5 March 2018 Revised 9 0ctober 2018 Accepted 23 November 2018

D) Check for updates

(C) Author(s) (or their employer(s)) 2019. Re-use permitted under CC BY. Published by BMJ.

${ }^{1}$ Department of Health, Ministry of Health, Nutrition and Indigenous Medicine, Colombo, Sri Lanka

${ }^{2}$ Division of Health Sciences, Department of Clinical Sciences, Collage of Health and Life Sciences, Brunel University, London, UK

${ }^{3}$ Health Economics Theme, Institute of Environment, Health and Societies, Brunel University, London, UK

Correspondence to Dr Priyanga

Diloshini Ranasinghe; priyangaran@yahoo.com, Priyanga.Ranasinghe@brunel. ac.uk

\section{ABSTRACT}

Introduction Evidence on the economic costs of physical inactivity and the cost-effectiveness of physical activity interventions in low-income and middle-income countries (LMICs) is sparse, and fragmented where they are available. This is the first review aimed to summarise available evidence on economics of physical activity in LMICs, identify potential target variables for policy, and identify and report gaps in the current knowledge on economics of physical activity in LMICs.

Methods and analysis Peer-reviewed journal articles of observational, experimental, quasi-experimental and mixed-method studies on economics of physical activity in LMICs will be identified by a search of electronic databases; Scopus, Web of Science and SPORTDiscus. Websites of WHO, the National Institute for Health and Care Excellence international, World Bank and reference lists of included studies will be searched for relevant studies. The study selection process will be a two-stage approach; title and abstract screen for inclusion, followed by a review of selected full-text articles by two independent reviewers. Disagreements will be resolved by consensus and discussion with a third reviewer. Data will be extracted using standardised piloted data extraction forms. Risk of bias will be critically appraised using standard checklists based on study designs. Descriptive synthesis of data is planned. Where relevant, summaries of studies will be classified according to type of economic analysis, country or country category, population, intervention, comparator, outcome and study design. Meta-analysis will be performed where appropriate. This protocol for systematic review is prepared according to the Preferred Reporting Items for Systematic review and Meta-analysis for Protocols -2015 statement.

Ethics and dissemination Ethical approval is not obtained as original data will not be collected as part of this review. The completed review will be submitted for publication in a peer-reviewed journal and presented at conferences.

PROSPERO registration number CRD42018099856.

\section{INTRODUCTION}

\section{Rationale}

Physical inactivity is a global health challenge, the fourth leading cause of mortality worldwide $^{1}$ and the key risk factor for non-communicable diseases (NCDs) such as cardiovascular diseases, cancer and diabetes. ${ }^{2}$ Almost a third

\section{Strengths and limitations of this study}

This is the first synthesis of literature on economics of physical activity in low-income and middle-income countries (LMICs).

- This study provides evidence-based recommendations for economic research practice on physical activity.

- This review presents evidence on the business case for physical activity interventions for decision making by policy makers in LMIC settings.

- This review could suffer from publication bias as it excludes studies that are not peer reviewed.

- This review focuses on studies written in the English language and could miss out on relevant literature published in other languages.

of the global health burden comprises NCDs which account for 1.4 billion disability-adjusted life years (DALYs). ${ }^{3}$ At present most of the global NCD burden is from low-income and middle-income countries (LMICs) (as defined by ${ }^{4}$ the World Bank, Classification 2017) ${ }^{4}$ and this burden is largely attributable to levels of physical inactivity in the population. ${ }^{56}$ Prevalence of physical inactivity in 2010 in upper middle-income countries was $25.4 \%$ $(19.1 \%-33.7 \%)$, followed by $16.8 \%$ (11.8\%$26.4 \%) \%$ ) in lower middle-income countries and $16.6 \%(11.8 \%-26.4 \%) \%)$ in low-income countries showing that large populations in $\mathrm{LMICs}^{7}$ are affected by physical inactivity and are therefore at risk of developing NCDs. The data available for the year 2004 show that 3.2 million deaths worldwide were due to physical inactivity of which 2.6 million were reported from LMICs. ${ }^{8}$ LMICs have experienced urbanisation, shift of occupations from agriculture to industrial practice and modernisation including automation, and this has resulted in increased levels of physical inactivity in these populations. ${ }^{9}$

Increasing physical activity and reducing the burden of NCDs is the main goal of public health policies in LMICs. ${ }^{10}$ It is a personal, corporate and government responsibility to 
reduce the economic burden of unhealthy lifestyles in LMICs. ${ }^{10}$ Economic analysis could uncover subtle characteristics of individual decision making on lifestyle choices, estimate the opportunity costs of not doing anything to improve population levels of physical activity and help evaluate what interventions work efficiently to achieve the goal of addressing the physical inactivity pandemic, particularly in LMICs. ${ }^{11}$ The current focus of promoting physical activity has shifted from the traditional education approach to environment and policy approaches. Economic perspectives and public health perspectives complement each other in promotion of physical activity. ${ }^{12}$ Economic perspectives inform the design of effective physical activity interventions (identifying economic barriers to physical activity) and the efficient allocation of resources-critical to the economies of LMICs. ${ }^{12}$

Our scoping exercise did not identify any published reviews that address the economics of physical activity in LMICs. Thus economic evidence on physical activity in LMICs could be limited or scattered. ${ }^{13}$ Therefore, we designed this review to address the following research questions: (1) What is the available evidence base and research gaps on the economics of physical activity in LMICs? (2) What is the focus of and methods for underpinning economic research on physical activity in LMICs? (3) What are the target variables and cost-effective interventions for physical activity policy in LMICs?

This review will add to the scientific literature, provide an overview of the economic evidence base of physical activity in LMICs and fill the gaps in the available evidence regarding this. Providing an up-to-date synthesis of the economic evidence base is an efficient way of highlighting current research practices and new findings to inform researchers, and formulation of cost-effective physical activity programmes and policies.

\section{Objectives}

The review will:

- Summarise available evidence on economics of physical activity in LMICs.

- Describe the focus and methods underpinning research on economics of physical activity in LMICs.

- Identify potential target variables and cost-effective interventions for physical activity in LMICs for policy formation.

- Identify and report gaps in research on economics of physical activity in LMICs to provide recommendations for the economic research agenda in LMICs.

\section{METHODS}

The methods for this review were informed by previous reviews of economic analyses of physical activity ${ }^{13-19}$ and are in line with recommendations on review of economic evidence. ${ }^{20-22}$ This will be based on the 'Preferred Reporting Items for Systematic Review and Meta Analyses Protocols (PRISMA-P)' Statement ${ }^{23}$ (online supplementary file 1).

\section{Eligibility criteria}

Economic studies on physical activity in LMICs will be included. Specifically, the following study types will be included: (1) Economic evaluations of physical activity interventions. (2) Economic burden of physical inactivity. (3) Cost of physical activity participation. (4) Demand for physical activity. (5) Economic correlates of physical (in) activity. Physical activity is defined as any bodily movement produced by skeletal muscles that require energy expenditure-including activities undertaken while walking, playing, carrying out household chores, travelling and engaging in recreational pursuits. ${ }^{24}$ Physical inactivity is considered as a lack of physical activity. ${ }^{25}$

Physical activity interventions will not be specifically defined for this review in order to identify any physical activity intervention in LMICs. However, studies of which an economic analysis was carried out along with the intervention will be included in this review. This strategy will enable consolidation of all available evidence on economics of physical activity related to physical activity interventions.

Eligibility criteria are determined by relevant elements of the population, intervention, comparator, outcome, study design (PICOS criteria). ${ }^{26}$ Interventions and comparators will be applicable only to intervention studies.

\section{Inclusion criteria}

- Study setting: Any setting of LMICs in accordance with the definition of LMIC by World Bank, Classification $2017 .^{4}$

- Population: Any population in any age group across the life course.

- Intervention: Any physical activity intervention in which the economic evaluation of the intervention has been carried out.

- Comparator: Normal routine, no intervention.

- Outcomes: (1) Cost-effectiveness ratio, quality-adjusted life years (QUALY), incremental cost-effectiveness ratio assessed as the outcomes of physical activity interventions. (2) Cost of physical (in) activity in terms of healthcare cost and/or productivity loss and/or total cost of physical inactivity. (3) Measures of association of any economic variable with physical activity are defined as the primary outcomes of this review.

- Study design: Observational studies (cohort, case control, cross-sectional); correlational studies, experimental studies including randomised controlled trials; quasi-experimental studies; natural experiments; and economic evaluation studies.

- Studies reported only in the English language.

\section{Exclusion criteria}

- Case reports, case series, letters to the editor, editorials, reviews, qualitative studies, unpublished theses, conference abstracts and any unobtainable texts.

- Studies published in a language other than English. 


\section{Search strategy}

The selection of databases was performed by a scoping review of methods of systematic reviews in the field. ${ }^{1317182728}$ We will search the following electronic databases to identify studies: Scopus (covers $100 \%$ MEDLINE coverage, $100 \%$ of EMBASE coverage and $100 \%$ of Compendex coverage ${ }^{29}$ ), Web of Science and SPORTDiscus. Websites of WHO, National Institute for Health and Care Excellence (NICE) international and World Bank will be searched for relevant studies. The reference lists of included studies will be searched for any relevant articles. Searches will include publications up to December 2017.

The search strategy was developed based on the scoping review that covered relevant reviews on economic studies on physical activity, ${ }^{10} 13-19$ reviews on physical activity ${ }^{27} 3031$ and reviews on economic evaluations. ${ }^{28} 3233$ The draft search strategy was then reviewed by subject experts and a subject liaison librarian to optimise the sensitivity and specificity of the search. Online supplementary file 2 shows a sample search strategy.

\section{Study selection}

A two-stage approach involving three independent reviewers will be used to select relevant papers.

At the first stage, two reviewers (PDR and NKA) will independently screen the titles and abstracts of identified papers. Disagreement will be resolved through discussions with a third reviewer (SP). We will include a study if in doubt about its inclusion. For example, if the country setting of a study is unclear in the title and abstract, we will take it forward to the next stage.

At the second stage, the full text of papers selected from stage 1, will be reviewed independently by two reviewers (PDR and NKA). Selected and excluded papers, with reasons, will be discussed by the two reviewers and a third reviewer (SP) at a consensus meeting and disagreement will be resolved by real time consensus. Corresponding authors will be contacted via email for clarification if needed. The study selection process will be presented in a PRISMA flow chart ${ }^{34}$ along with the reasons for the exclusion of studies.

\section{Data management and extraction}

Endnote X7 software will be used to manage the search results. Data extraction will be performed using a standardised pilot-tested data extraction form developed based on relevant data extraction forms from relevant reviews. ${ }^{173536}$ The adaptation process involved matching the review objectives with the available data extraction forms. Data from the final selected full-text articles will be extracted by one reviewer (PDR). To ensure quality of data extraction, a second reviewer (NKA) will independently extract data from a random selection of $50 \%$ of the final articles. Disagreements between the two reviewers will be discussed and resolved through discussions with the third reviewer (SP). Any unresolved disagreements will be reported in the final report. Online supplementary file 3 shows the draft data extraction form. Data will be extracted for the following items: general information, characteristics of the study, characteristics of the population/condition/intervention, data sources/data analysis/ outcomes, conclusions and the way forward suggested by the authors, challenges and quality assessment.

\section{Risk of bias and quality assessment}

Risk of bias will be assessed by a one reviewer (PDR) for all the selected articles using standard checklists based on the study design. A second reviewer (NKA) will also independently assess the risk of bias on $50 \%$ of the randomly selected articles included.

Checklists are considered a reliable means of ensuring that all studies included are critically appraised in a standard way. ${ }^{26}$ These will assess the information bias which could occur due to the methods, study design, data collection, data analysis and interpretation, and selection bias, where relevant. The most appropriate checklists for each expected study design for risk of bias assessment were selected based on relevant literature in this area ${ }^{15} 1736-40$ and by consensus with all three reviewers (online supplementary file 4). Risk of bias will be assessed using the following tools:

- Economic evaluation studies

The Drummond checklist ${ }^{41}$ which consists of 35 items will be used. It mainly assesses the quality of the study in relation to methods, study design, data collection, data analysis and interpretations. If an economic evaluation study is based on a decision analytical model, the Phillips ${ }^{42}$ checklist will be used in addition.

- Cost of illness studies

The Larg \& Moss checklist ${ }^{43}$ will be used to assess the quality of the study. It would assess the possible biases related to the analytical framework: what costs should have been measured, methodology and data; how well were resources used and productivity losses measured including representativeness of data for the study population, analysis and reporting. Both internal validity and external validity will be appraised.

- Studies on correlation or association

The checklist recommended by $\mathrm{NICE}^{44}$ will be used. Risk of bias related to sample selection, method of selection of exposure/comparison group, outcomes and analysis will be assessed. Both internal and external validity will be appraised.

Quality grading will be given to each included study based on the overall quality of the study. Grading for internal validity and external validity will be reported separately, where relevant. The grading process will follow the NICE recommendations ${ }^{37}$ (figure 1):

- Good quality $(++)$ : almost all checklist criteria have been fulfilled; where they have not been fulfilled the conclusions are very unlikely to alter.

- Moderate quality (+): some of the checklist criteria are fulfilled; where they have not been fulfilled or not adequately described, conclusions are unlikely to alter. 


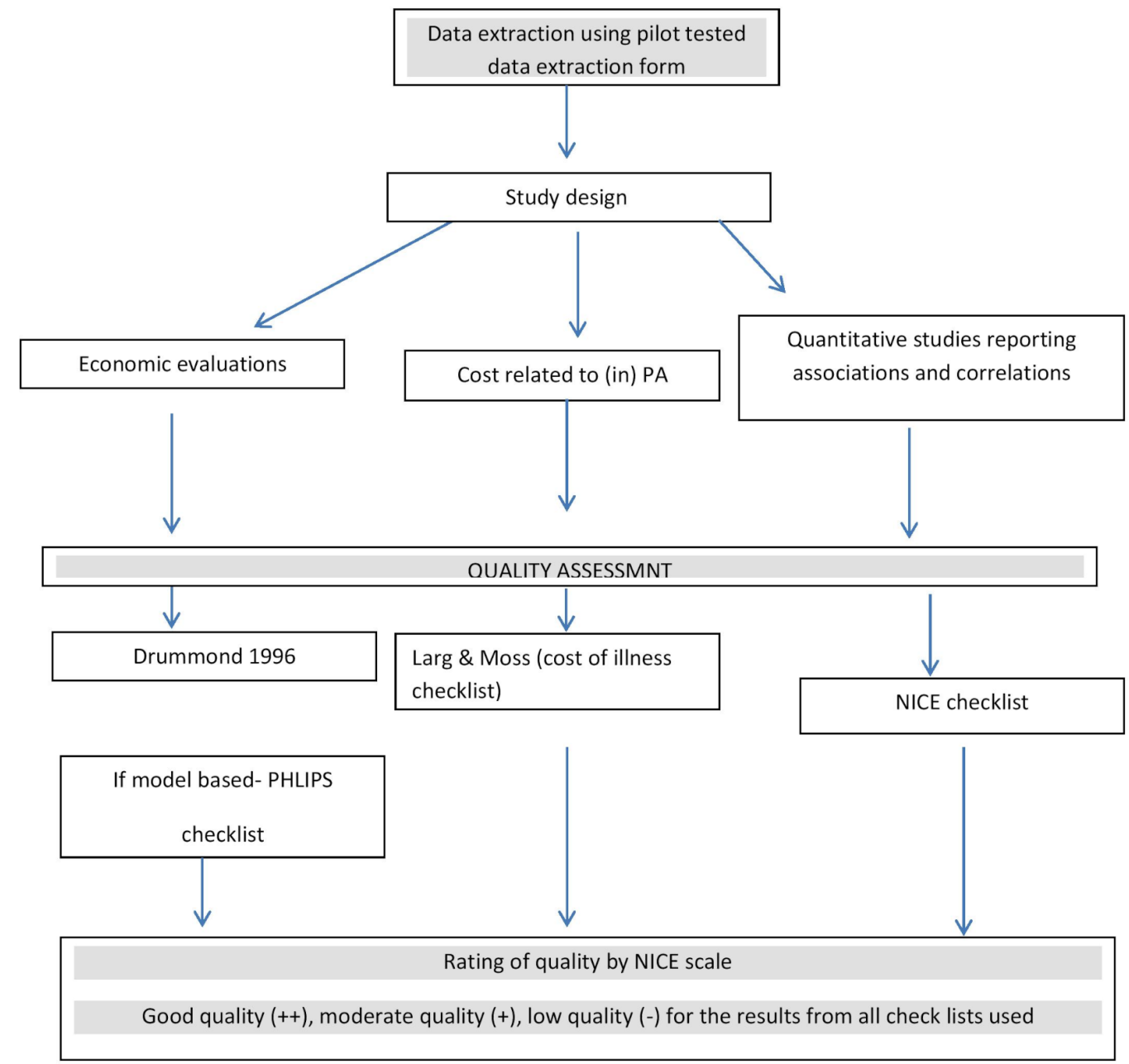

Figure 1 Economics of physical activity (PA) in low-income and middle-income countries (LMICs) - plan for data extraction and the quality assessment process. NICE, National Institute for Health and Care Excellence.

- Low quality (-): few or no checklist criteria are fulfilled, and conclusions are likely or very likely to alter.

- The quality of the included studies will be reported and critically appraised for each study. However, studies will not be excluded based on quality, as this review is particularly aimed to capture the evidence base on economics of physical activity in LMICs and appraise the methods underpinning the evidence base.

\section{Data synthesis and reporting}

A descriptive synthesis of data is planned due to the expected heterogeneity of the studies. Summaries of studies will be classified according to type of economic analysis, country or country category, characteristics of the study by PICOS criteria (where applicable); population, intervention, comparator, outcome, study design and quality of studies to describe the overview of available economic evidence base of physical activity in LMICs. Where the numbers permit, summaries of the studies will be categorised based on the focus of the study; economic burden of physical inactivity, economic analysis of physical activity interventions, economic correlates of physical activity including association of physical activity and cost (healthcare cost and/or productivity loss). Where appropriate, we will conduct a meta-analysis to quantify the effect sizes. Such analyses will adjust for between-study heterogeneity using random-effects models. The I-squared $\left(\mathrm{I}^{2}\right)$ statistic will be used to evaluate the heterogeneity related to the findings of the papers. Quantitative analysis will be conducted using STATA V.13 software.

We will report the number of studies where data extraction items were not applicable, as an indicator of the quality of reporting. Author-stated limitations of included studies and recommendations for future research and policy will be presented. This review will be reported in accordance with the PRISMA 2009 statement. ${ }^{34}$ The study selection process will be illustrated by a flow diagram that will include the reasons for exclusion of studies at each stage. The search strategy, data extraction forms and quality assessment tools will be published as online supplementary files. This protocol will not be amended and any changes will be described and discussed in the final report. 


\section{Study status}

A scoping review of economics of physical activity in LMICs was carried out in November 2017 to inform the methods of the current review. It identified systematic reviews conducted on economics of physical activity and the gaps in knowledge. It has not been submitted for publication. A review of economics of physical activity in LMICs was designed and methods were developed in December 2017. The search strategy was piloted in January 2018 and the protocol was developed in February 2018. The first submission of the protocol for peer review was on 1 March 2018. Data collection will be commenced upon approval of the protocol for publication and will be completed in 2 months.

\section{Patient and public involvement}

Patients and public are not directly involved in this study as original data will not be collected.

Best practice guidelines to conduct systematic reviews ${ }^{33}$ will be followed.

\section{DISCUSSION}

The level of physical inactivity of the population in LMICs is likely to increase giving rise to a multitude of public health and economic consequences, including rising healthcare costs due to increased NCD-related treatments, increased DALYs and productivity losses. Economic research on physical activity from the perspective of LMICs should therefore be considered a priority. This is because increased physical inactivity leads to uncontrolled healthcare needs in LMICs, where resources (including that for healthcare) are limited and often shrinking in real terms. Pooling of the available research evidence on economics of physical activity in LMICs will thus reveal the current knowledge on economics of physical activity in LMICs, thereby guiding the future of national, regional and local policies around physical activity.

This will be the first study to review the evidence base on economics of physical activity in the context of LMICs which can be considered as the main strength of this study. This will identify gaps in knowledge to support future studies in this area. However, we will only include those studies that are published as peer-reviewed articles and are published in the English language. Thus, possibility of publication bias and language bias cannot be excluded. Furthermore, by assessing and reporting the quality of included studies using standard quality assessment checklists, the possibility of reporting bias of this review can be minimised.

WHO is in the process of drafting the "Global action plan on physical activity (GAPPA) to be implemented from 2018 to $2030{ }^{\prime} .{ }^{45}$ This is a timely study as the evidence from this research will provide useful information for implementation and prioritise the actions of GAPPA in LMICs. Evidence from this research will be useful for policy makers and stakeholders dealing with physical activity promotion at individual, local, national, regional and global levels, with special reference to LMICs. Further, this review will be an important base for a research agenda on economics of physical activity in LMICs. This will be a useful guide for researchers to design research on economics and physical activity with sound methodology, based on the research needs of LMICs. Furthermore, information gathered from this research will guide funding agencies for effective allocation of resources.

Contributors SP and NKA developed the idea for the review with inputs from PDR. PDR wrote the first draft. SP and NKA revised the protocol. NKA will act as guarantor of the review.

Funding The authors have not declared a specific grant for this research from any funding agency in the public, commercial or not-for-profit sectors.

Competing interests None declared.

Patient consent for publication Not required.

Provenance and peer review Not commissioned; externally peer reviewed.

Open access This is an open access article distributed in accordance with the Creative Commons Attribution 4.0 Unported (CC BY 4.0) license, which permits others to copy, redistribute, remix, transform and build upon this work for any purpose, provided the original work is properly cited, a link to the licence is given, and indication of whether changes were made. See: https://creativecommons.org/ licenses/by/4.0/.

\section{REFERENCES}

1. World Health Organization. Global recommendations on physical activity for health. Geneva: World Health Organization, 2010.

2. World Health Organization. Physical activity [Fact Sheet - Media centre]. 2017 http://www.who.int/mediacentre/factsheets/fs385/en/ (Accessed 6 Feb 2018).

3. Murray CJ, Barber RM, Foreman KJ, et al. Global, regional, and national disability-adjusted life years (DALYs) for 306 diseases and injuries and healthy life expectancy (HALE) for 188 countries, 1990-2013: quantifying the epidemiological transition. Lancet 2015;386:2145-91.

4. Bank W. World bank country and lending groups: country classification. 2017 https://datahelpdesk.worldbank.org/ knowledgebase/articles/906519-world-bank-country-and-lendinggroups (Accessed 1st Dec 2017).

5. Abegunde DO, Mathers CD, Adam T, et al. The burden and costs of chronic diseases in low-income and middle-income countries. Lancet 2007;370:1929-38.

6. Hallal PC, Andersen LB, Bull FC, et al. Global physical activity levels: surveillance progress, pitfalls, and prospects. Lancet 2012;380:247-57.

7. WHO. Prevalence of insufficient physical activity among adults, data

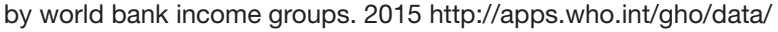
view.main.2487? lang=en (Accessed 29th Jan 2018).

8. WHO. Global health risks: mortality and burden of disease attributable to selected major risks. ISBN 978924156387 1. 2009. Retrieved from http://www.who.int/healthinfo/global_burden_ disease/GlobalHealthRisks_report_full.pdf (Accessed 25th Jan 2018).

9. Atkinson K, Lowe S, Moore S. Human development, occupational structure and physical inactivity among 47 low and middle income countries. Prev Med Rep 2016;3:40-5.

10. Oldridge NB. Economic burden of physical inactivity: healthcare costs associated with cardiovascular disease. Eur J Cardiovasc Prev Rehabil 2008;15:130-9.

11. Islam SM, Purnat TD, Phuong NT, et al. Non-communicable diseases (NCDs) in developing countries: a symposium report. Global Health 2014; $10: 81$.

12. Sturm R. Economics and physical activity: a research agenda. Am J Prev Med 2005;28:141-9.

13. Ding D, Kolbe-Alexander T, Nguyen B, et al. The economic burden of physical inactivity: a systematic review and critical appraisal. $\mathrm{Br} J$ Sports Med 2017;51:1392-409.

14. Barte JCM, Wendel-Vos GCW. A systematic review of financial incentives for physical activity: the effects on physical activity and related outcomes. Behav Med 2017;43:79-90.

15. Abu-Omar K, Rütten A, Burlacu I, et al. The cost-effectiveness of physical activity interventions: a systematic review of reviews. Prev Med Rep 2017;8:72-8. 
16. Brown V, Zapata Diomedi B, Moodie M, et al. A systematic review of economic analyses of active transport interventions that include physical activity benefits. BMJ Open 2016;45.

17. Pavey TG, Taylor AH, Fox KR, et al. Effect of exercise referral schemes in primary care on physical activity and improving health outcomes: systematic review and meta-analysis. BMJ 2011;343:d6462.

18. Campbell F, Holmes M, Everson-Hock E, et al. A systematic review and economic evaluation of exercise referral schemes in primary care: a short report. Health Technol Assess 2015;19:1-110.

19. Suhrcke M, Boluarte TA, Niessen L. A systematic review of economic evaluations of interventions to tackle cardiovascular disease in lowand middle-income countries. BMC Public Health 2012;12:2.

20. Kaltenthaler E, Tappenden P, Paisley S. Reviewing the evidence to inform the population of cost-effectiveness models within health technology assessments. Value Health 2013;16:830-6.

21. Zechmeister-Koss I, Schnell-Inderst P, Zauner G. Appropriate evidence sources for populating decision analytic models within health technology assessment (HTA): a systematic review of HTA manuals and health economic guidelines. Med Decis Making 2014;34:288-99.

22. NICE. The guideline manual process and methods (PMG6): NICE, 2012.

23. Moher $D$, Shamseer L, Clarke M, et al. Preferred reporting items for systematic review and meta-analysis protocols (PRISMA-P) 2015 statement. [Research Support, Non-U S Gov't Research Support, U S Gov't, P H S]. Syst Rev 2015;4:2046-4053.

24. WHO. Physical Activity. [Fact sheet]. 2018 http://www.who.int/en/ news-room/fact-sheets/detail/physical-activity (Accessed 15th Jan 2018).

25. WHO. Global Strategy on Diet, Physical activity and Health. 2018. Retrieved from https://www.who.int/dietphysicalactivity/pa/en/ (Accessed 6th Oct 2018).

26. University of York. Systematic reviews: CRD's guidance for undertaking reviews in health care. York: University of York, 2009.

27. Milton K, Macniven R, Bauman A. Review of the epidemiological evidence for physical activity and health from low- and middleincome countries. Glob Public Health 2014;9:369-81.

28. Nosratnejad S, Rashidian A, Dror DM. Systematic review of willingness to pay for health insurance in low and middle income countries. PLoS One 2016;11:e0157470.

29. Burnham JF. Scopus database: a review. Biomed Digit Libr 2006;3:1742-5581

30. Lachat C, Otchere S, Roberfroid D, et al. Diet and physical activity for the prevention of noncommunicable diseases in low- and middle-income countries: a systematic policy review. PLoS Med 2013;10:e1001465.

31. Hunter RF, Christian H, Veitch J, et al. The impact of interventions to promote physical activity in urban green space: a systematic review and recommendations for future research. Soc Sci Med 2015;124:246-56.
32. Sutton L, Karan A, Mahal A. Evidence for cost-effectiveness of lifestyle primary preventions for cardiovascular disease in the Asia-Pacific Region: a systematic review. Global Health 2014;10:79.

33. Santatiwongchai $B$, Chantarastapornchit V, Wilkinson T, et al. Methodological variation in economic evaluations conducted in low- and middle-income countries: information for reference case development. PLoS One 2015;10:e0123853.

34. Moher D, Liberati A, Tetzlaff J, et al. Preferred reporting items for systematic reviews and meta-analyses: the PRISMA statement. [Guideline Research Support, Non-U S Gov't]. J Clin Epidemiol 2009;62:1006-12.

35. Pokhrel S, Quigley MA, Fox-Rushby J, et al. Potential economic impacts from improving breastfeeding rates in the UK. [Research Support, Non-U.S. Gov't]. Arch Dis Child 2015;100:334-40.

36. Murthy S, John D, Godinho IP, et al. A protocol for a systematic review of economic evaluation studies conducted on neonatal systemic infections in South Asia. Syst Rev 2017;6:252.

37. Aminde LN, Veerman L. Interventions for the prevention of cardiovascular diseases: a protocol for a systematic review of economic evaluations in low-income and middle-income countries. BMJ Open 2016;6:e013668.

38. Ruifrok AE, Rogozinska E, van Poppel MN, et al. Study protocol: differential effects of diet and physical activity based interventions in pregnancy on maternal and fetal outcomes--individual patient data (IPD) meta-analysis and health economic evaluation. Syst Rev 2014;3:131.

39. Gc V, Wilson EC, Suhrcke M, et al. Are brief interventions to increase physical activity cost-effective? A systematic review. Br J Sports Med 2016;50:408-17.

40. Tonmukayakul U, Calache $\mathrm{H}$, Clark R, et al. Systematic review and quality appraisal of economic evaluation publications in dentistry. $J$ Dent Res 2015;94:1348-54.

41. Drummond MF, Jefferson TO. Guidelines for authors and peer reviewers of economic submissions to the BMJ. BMJ 1996;313:275-83.

42. Philips Z, Ginnelly L, Sculpher M, et al. Review of guidelines for good practice in decision-analytic modelling in health technology assessment. Health Technol Assess 2004;8:1-158.

43. Larg A, Moss JR. Cost-of-illness studies: a guide to critical evaluation. [Research Support, Non-U S Gov't]. Pharmacoeconomics 2011;29:653-71.

44. National Institute for Health and Excellence (NICE). Methods for the development of NICE public health guidance (third edition), Appendix G Quality appraisal checklist-quantitative studies reporting correlations and associations. 2012 https://www.nice.org.uk/ process/pmg4/chapter/appendix-g-quality-appraisal-checklistquantitative-studies-reporting-correlations-and (Accessed 2 nd Jan 2018).

45. World Health Organization. Non communicable diseases and their risk factors, governance: Development of draft global action plan to promote physical activity, 2018. 